\title{
Stability of lauric acid at high pressure studied by Raman spectroscopy and picosecond acoustics
}

\author{
Sayed Mohamed Baqer Albahrani, Guilhem \\ Simon, Simon Ayrinhac, Michel Gauthier, \\ Frederic Decremps, Isabelle Lisiecki, Salvatore \\ Constanzo, Philippe Colomban
}

\begin{abstract}
Lauric acid is commonly used as a coating agent which efficiently protects against oxidation and/or coalescence a set of inorganic nanocrystals obtained by chemical process. Its stability under pressure is likely to be informative on the stability and ordering of compressed supercrystals of nanocrystals. Therefore the elastic behaviour of lauric acid submitted to high pressures up to $25 \mathrm{GPa}$ is studied. This elastic behavior has been probed by two complementary in situ techniques at high pressure : Raman spectroscopy and picosecond acoustics. Comparison between pressure-induced transformations as observed with the two techniques suggests that lauric acid remains elastically stable above $2 \mathrm{GPa}$ up to $25 \mathrm{GPa}$.
\end{abstract}

Keywords fatty acid; lauric acid ; supracrytals ; high pressures ; Raman spectroscopy; picosecond acoustics

\section{Introduction}

Ordered 3D assemblies of metallic nanocrystals (NCs), called supercrystals, exhibit interesting properties in phononics, optics, magnetism and so on [1-5]. Among this class of nanomaterials, Cobalt (Co) supracrystals are promising materials for many applications such as ultrahigh density magnetic data storage, electronics and sensors [6-10]. One of the prerequisite to create supercrystals is the use of highly stable NCs characterized by a narrow size distribution. For example, lauric acid coated Co NCs with a mean diameter and size distribution of $7.1 \mathrm{~nm}$ and $11 \%$ respectively are synthesized using chemical reduction of Co salts, like $\mathrm{Co}(\mathrm{AOT})_{2}$ and lauric acid as coating agent and they are used as the building units of long-range ordered fcc

Send offprint requests to: Guilhem Simon

S.M.B Albahrani

Sorbonne Université, CNRS, Museum National d'Histoire Naturelle, IRD, Institut de Minéralogie, de Physique des Materiaux et de Cosmochimie, IMPMC, F-75005 Paris, France

Sorbonne Université, CNRS, Molécules Nanomatériaux, Interactions, Réactivité et Spectroscopies, MONARIS, F-75005 Paris, France 
supercrystals [11,12]. Lauric acid (or dodecanoic acid) is a saturated fatty acid, with a 12-carbon atom chain, found mostly in coconut oil (47\%) which is a common vegetable oil. When used in Co supracrystals, these organic molecules fulfill two main functions : (1) they prevent efficiently the oxidation of the cobalt as well as the coalescence between the particles ; (2) they act like mechanical nano springs holding together the NCs in a regular network $[13,4]$. Thus, they not only ensure the stability of both the NCs and their 3D organisations but they also play a key role in the structural characteristics, and the properties of the supercrystal. The self-assembly and the stability in supracrystals results from a complex interparticle interaction forces including: interactions between organic chains, interactions between Co nanospheres and interactions between Co NCs and the chains. These interactions are far from being understood [1]. These interactions can be explored by submitting these systems to high pressure or high temperature conditions. It should be noted however that pressure permits to increase more efficiently the interactions. Under high pressure, instabilities can lead to phase transitions, for instance from face-centered-cubic (fcc) structure to hexagonal close packed (hcp) "super"structure, or also can favour an increasing/decreasing of the coherence length [14].

Considering that the Co nanospheres are regularly ordered in a fcc superlattice in an idealized case within a supracrystal [10], the coating agent occupies more than $50 \%$ of the volume of the sample (as depicted in the supplemental material of Ref. [4]). As mentioned below, the coating agent may strongly contribute to determine the physical properties of the overall system, especially if consider that the stability of crystalline cobalt lattice inside a nanoparticle is maintained up to $100 \mathrm{GPa}$ [15]. So the behaviour of the lonely lauric acid submitted to extreme conditions of pressure is worth being investigated. Unlike the reported effect of temperature on lauric acid used as a phase material for energy storage [16], no results on the effect of pressure is reported in literature.

In the current study, we report a high-pressure study of polycrystalline lauric acid in order to identify the eventual phase transformations occurring in the range of pressure between ambient up to $25 \mathrm{GPa}$. The choice of a polycrystalline structure is motivated by the fact that the lauric acid chains within Co supracrystals are distributed in a random and disordered manner. Polycrystalline lauric acid can thus be considered equivalent to these chains, and be used as a good model to describe their random orientation, provided that the volume probed by our techniques is superior to that of a monocrystalline domain. The investigation of phase transformations is performed with two complementary in situ techniques: Raman scattering technique and picosecond acoustics technique. The latter is equivalent to time-resolved Brillouin spectroscopy, and it exploits the sound velocity as a sensitive tool to probe the phase changes in material [17].

Many data have been reported in literature on the elastic properties of liquid fatty acids (see for example [18] or [19]), but to our knowledge, this is the first time that elastic properties are measured at high pressure in a solid fatty acid. The lack of results in these extreme conditions are likely due to the difficulty encountered during the measurements of ultrasonic propagation in solid fats (pure solid triglycerides) which tends to form voids upon cooling [20].

Phase transitions were, nevertheless, detected in other solid fatty acids : in palmitic acid [21], in oleic acid [22], or in linoleic acid [23]. However, these phase transitions are detected only in Raman scattering. 


\section{Experimental details}

\subsection{Sample preparation}

The generating pressure apparatus is a membrane diamond anvil cell (mDAC, Le Toullec type) equipped with two diamonds with a culet diameter of $400 \mu \mathrm{m}$.

A small piece of aluminum is firstly mashed between the two culets to obtain a metallic transducer, with a thickness around $150 \mathrm{~nm}$. A small piece of solid lauric acid (from Sigma-Aldrich, purity $98 \%$ ) is then smashed between the culets inside a hole drilled in a metallic gasket (using laser micro-machining device). Then the $\mathrm{DAC}$ is put into an oven around $100^{\circ} \mathrm{C}$ so lauric acid is melted before the complete closure of the DAC to ensure that the hole is fully filled.

The orientation of lauric acid crystal is not controlled and is supposed to be polycristalline. Two ruby microspheres are introduced in the sample chamber, in order to monitor the pressure thanks to the ruby fluorescence Raman pressureinduced shift [24].

\subsection{Picosecond acoustics}

The picosecond acoustics technique is an optical pump-probe technique used to probe the acoustic response of a sample at the nanosecond scale [25]. The experimental setup is the same as the one used in previous experiments by some of the authors of the current paper, and a detailed description is presented therein [26]. A femtosecond Ti:sapphire laser source emits an infrared beam at $\lambda_{0}=800 \mathrm{~nm}$ that is splitted into pump and probe beams. The pump laser beam is focused on the aluminum metallic transducer on which lauric acid sample lies, whereas the probe laser arrives to the opposite surface of the sample after passing through a delay line in order to perform time-varying measurements. The local heating of the metallic transducer creates a strain wave that propagates inside the sample. The interference between the probe beam reflected by the static interfaces of the sample and reflected by the the propagating strain wave produce oscillations in the signal. These time-resolved oscillations are called Brillouin oscillations since they are analog to the frequency-resolved Brillouin scattering [27].

At normal incidence, the frequency $f$ of these oscillations is given by the formula

$$
f=\frac{2 n v}{\lambda_{0}},
$$

where $n$ is the refractive index of the transparent medium at the wavelength $\lambda_{0}$ and $v$ is the longitudinal sound velocity. The probed frequency of the phonon inside the wavepacket of the propagating strain wave and the period $T$ of the Brillouin oscillations are related by the obvious relation $T=1 / f$.

\subsection{Raman scattering}

Raman scattering is an inelastic process in which one incident photon at the frequency $\omega_{p h}$ interacts with a vibrational quantum of the probed material and give 


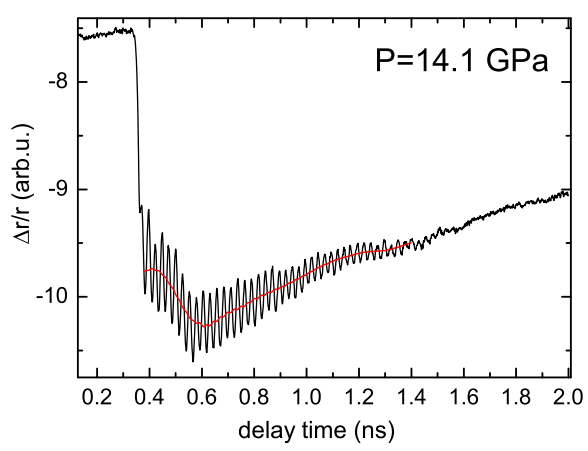

Fig. 1 Typical signal (raw data) of relative reflectivity variations in lauric acid at $\mathrm{P}=14.1 \mathrm{GPa}$ and ambient temperature. Brillouin oscillations are extracted after substraction of a base line (red curve).

rises to a scattered photon at the frequency $\omega_{s}=\omega_{p h} \pm \omega_{v}$. The plus and minus signs stand for anti-Stokes and stokes processes respectively.

The Raman spectra were collected using a HR800 LabRam setup from Horiba Scientific equipped with a cooled charge coupled device (CCD) detection system. Excitation was done with the green line of Argon Laser at $\lambda_{0}=514.5 \mathrm{~nm}$. The laser power at the focus point was $50 \mu \mathrm{W}$. A long working distance microscope objective $(\times 50$, Mitutoyo) with a focal distance of $20.5 \mathrm{~mm}$ and numerical aperture (NA) of 0.24 was used to focus the laser and collect the scattered light from the sample. The spot size was about $50 \mu \mathrm{m}^{2}$ at the focus point and a confocal hole was used to avoid scattering contribution of the diamonds. The Raman setup was equipped with volume Bragg filters from Optigrate allowing low wavenumber measurements down to $10 \mathrm{~cm}^{-1}$ with high throughput.

\section{Results and discussions}

\subsection{Picosecond acoustics}

A typical signal of the relative reflectivity variations in lauric acid at ambient temperature and high pressure is shown in Fig. 1. The damping of Brillouin oscillations is due to both acoustic attenuation of the strain wave at the frequency $f$ and optical absorption of the probe at the $\lambda_{0}$ wavelength. The analysis of the damping is rather complicated since it has to take into account acoustic diffraction, coherence time and depth of the probe light laser pulse [27]. The steep change observed around $0.36 \mathrm{~ns}$ corresponds to the arrival of the propagating strain to the surface of the transducer.

Picosecond acoustics permits an in-depth resolution in transparent materials. It can be used to determine elastic inhomogeneities [28] or to probe grains in a polycristalline sample if those are large enough $[29,30]$. After subtraction of the base line, short time Fourier transform (STFT) is performed on the signal, with a normalization of the amplitude (Hanning window, $0.26 \mathrm{~ns}$ width). The result is shown in Fig. 2. The steep variations of frequency are probably due to the polycristallinity of the sample. Here in the STFT, two domains are visible with 


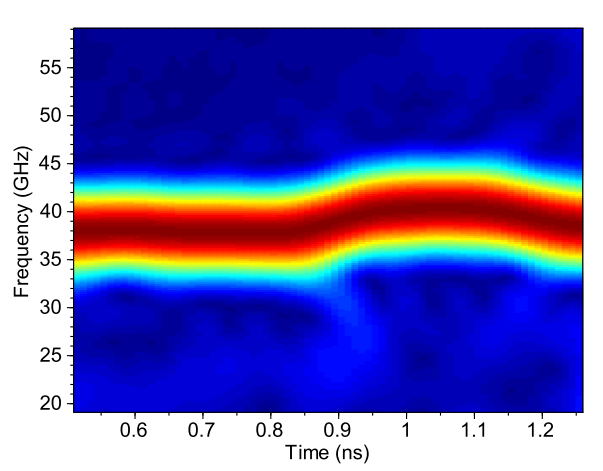

Fig. 2 Frequency as a function of time obtained by a short time Fourier transform (STFT) of the signal shown in Fig. 1 after subtraction of the background and normalization of the amplitude. See text for details. Color scale range from low signal (blue) to hig signal (red).

different frequencies of 38.0 and $40.4 \mathrm{GHz}$. The size of the different domains is strongly dependent of their localization within the sample.

The frequency of the Brillouin oscillations $f$ as a function of pressure for solid lauric acid is shown in the Fig. 3 up to $25 \mathrm{GPa}$. Period measurements from Brillouin oscillation are very accurate (the relative error is around $0.1 \%$ ) so the data dispersion is rather to be attributed to pressure gradients inside the sample, as well as the probable polycristallinity of the lauric acid. Measurements were made with increasing, and then with decreasing pressure. The reproducibility of the frequencies demonstrates the reversibility of the transformations induced by pressure. The increase in frequency is related to the increase of longitudinal sound velocity $v$ and refractive index $n$ through the Eq. 1 . Sound velocity $v$ is very sensitive to detect modifications in elastic properties varying $\mathrm{P}, \mathrm{T}$ conditions and hence to detect phase transitions ??. A continuous and smooth increase in $v$ claims that the compound remains elastically stable above $2 \mathrm{GPa}$ up to $25 \mathrm{GPa}$.

The inset in Fig. 3 shows an estimate of the pressure gradient $\Delta \mathrm{P}$ evolution with pressure level in the DAC. $\Delta \mathrm{P}$ was roughly estimated with the formula $\Delta P=$ $\frac{1}{2}\left|P_{2}-P_{1}\right|$, where $\mathrm{P}_{1}$ and $\mathrm{P}_{2}$ are pressures measured with two ruby microspheres placed in two opposite sides of the sample (separated at a distance of around $200 \mu \mathrm{m}$ ). The pressure gradients increase as the pressure level increases, confirming the presence of some non-hydrostaticity within the sample at higher pressures. It can be noticed by the way that the value of $\Delta \mathrm{P}$ permits a direct comparison of lauric acid with many other pressure transmitting media (PTM) studied in literature [31]. These comparisons show that lauric acid can be considered as a "soft" PTM : it exhibits less non-hydrostatic effects than Iso-n-pentane mixtures, and it solidifies in a comparable way to that of liquids or oils.

Miles et al. [32] have reported measurements of the velocity of ultrasound as a function of temperature in a number of predominantly solid animal and vegetable fats. Sound velocity values range between 1990 and $2080 \mathrm{~m} \cdot \mathrm{s}^{-1}$ in solid phase at $242 \mathrm{~K}$. This range of velocities combined with a refractive index around 1.5 yields an estimate of $f=7.5 \mathrm{GHz}$ at ambient pressure, in agreement with the measurement obtained at the lowest pressure point shown in Fig. 3. Furthermore, by using the value of density of lauric acid at ambient temperature and pressure, 


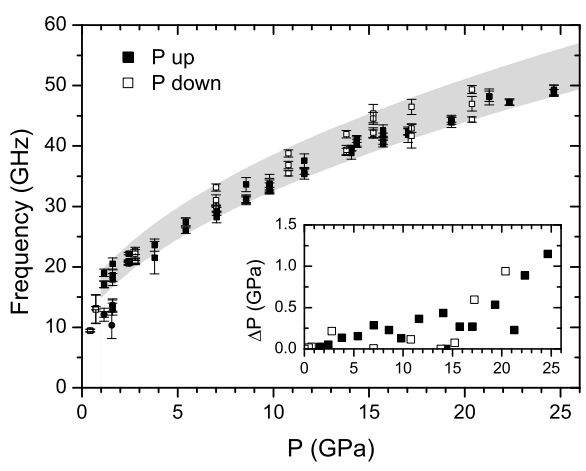

Fig. 3 Brillouin frequency in solid lauric acid at ambient temperature, as a function of pressure upstroke (filled squares) and downstroke (empty squares). The frequency values and error bars come from STFT analysis of the signals. The dispersion of the frequencies (shaded area) above $1 \mathrm{GPa}$ is mainly due to the sample polycrystallinity. Between 1 and $2 \mathrm{GPa}$ (filled circles) the signal contains low-frequency Brillouin oscillations, which can be attributed to a reminiscent part of the low-pressure phase. (Inset) Rough estimate of the pressure gradient $\Delta \mathrm{P}$ with two ruby microspheres, which indicates the increase of the pressure gradient inside the sample.

$\rho_{0}=1007 \mathrm{~kg} / \mathrm{m}^{3}$ [33], we can estimate the bulk modulus of lauric acid in the same conditions by using $B_{0}=\rho_{0} v_{0}^{2}=4 \mathrm{GPa}$.

\subsection{Raman scattering}

The assignments of the Raman modes can be done with the help of literature data on other fatty acids such as palmitic acid [21]. Since lauric acid samples used in this study are polycrystalline, all the vibrational modes should be observed. A comprehensive vibrational study of fatty acids $(\mathrm{C}=9,12,14,16,23$ and 26) have been made much earlier at room and low temperature [34-36]. These compounds, consisting of a carboxylic head connected to a zig-zag paraffinic chain ended with a $\mathrm{CH}_{3}$ group, have the peculiarity fo form dimers stabilized by a strong $\mathrm{H}$-bond between two adjacent carboxylic heads. Among these acids, palmitic acid (C16 hexadecanoic acid with $\left(\mathrm{CH}_{2}\right) 14$ chain) and stearic acid (i.e. $\mathrm{C} 18$ octadecanoic acid with $\left(\mathrm{CH}_{2}\right) 16$ chain) have already been studied by Raman spectroscopy in a DAC at room temperature $[37,23]$. Four phase transitions at $\simeq 1,6,12$ and 16 GPa have been identified by Raman spectoscopy in palmitic acid [37] and detailed analysis of stearic acid crystals pointed out that 3 phases can be identified below $4 \mathrm{GPa}$ [21].

The raw data spectra, and the associated bands, are shown in Figs. 4-7. The lines in wavenumber vs. pressure graphs are guides for the eyes : solid lines are used for major bands and dot or dashed lines are used for minor bands. Figs. 4-7 show the spectra recorded up to $\sim 19 \mathrm{GPa}$ in the spectral range allowed by DAC, namely low (20-400 $\left.\mathrm{cm}^{-1}\right)$, medium (800-1200 and 1400-1700 $\mathrm{cm}^{-1}$ ) and high (2700-3200 $\left.\mathrm{cm}^{-1}\right)$ wavenumber ranges for the lauric acid. Many runs have been made and thus the spectra recorded in the different windows have been recorded independently. Different pressures are also obtained depending on the studied areas in the DAC chamber. In the low wavenumber range (Fig. 4 (a)), two strong bands at 60 and $200 \mathrm{~cm}^{-1}$ are observed at ambient pressure (noted $0 \mathrm{GPa}$ ). The first mode $\left(60 \mathrm{~cm}^{-1}\right)$ is assigned to a lattice mode involving the O-HO bond be- 


\begin{tabular}{lll}
\hline Fatty acid (chain) & Transitions (GPa) & Ref. \\
\hline Lauric $\left[\left(\mathrm{CH}_{2}\right)_{10}\right]$ & $1,5,12$ & This work \\
Palmitic $\left[\left(\mathrm{CH}_{2}\right)_{14}\right]$ & $1,6,12,16$ & {$[37]$} \\
Stearic $\left[\left(\mathrm{CH}_{2}\right)_{16}\right]$ & $2.4,3.3$ & {$[21]$} \\
\hline
\end{tabular}

Table 1 Comparison between the pressure-induced phase transitions observed in lauric acid via Raman spectroscopy, and those reported in literature on other fatty acids.

tween two adjacent chains within a dimer and also to the CCC chain. The second mode $\left(200 \mathrm{~cm}^{-1}\right)$ is assigned to a combination of the CCC chain bending with the OO stretching mode of the H-bonded dimer [34]. The rather large broadness of these bands indicates some orientational disorder, as expected for low wavenumber modes of a compound close to its melting temperature $(316.2 \mathrm{~K})[38]$. In the medium wavenumber range, several bands are observed (Fig. 5 (a)). According to Ref. [35], the doublet at 890 and $905 \mathrm{~cm}^{-1}$ is assigned to $\rho\left(\mathrm{CH}_{2}\right)$ rocking modes, and the triplet at $1060-1075-1120 \mathrm{~cm}^{-1}$ is assigned to $\nu \mathrm{C}-\mathrm{C}$ stretching modes, and the massif between 1400 and $1500 \mathrm{~cm}^{-1}$ corresponds to a $\delta\left(\mathrm{CH}_{2}\right)$ scissoring mode, plus a minor contribution of the $\mathrm{CH}_{3}$ mode.

A phase transition takes place just after application of the pressure, at around $1 \mathrm{GPa}$. Indeed, the spectrum recorded at this latter pressure reveals more lattice mode components compared to the one obtained at ambient pressure ( 8 instead of 3 below $150 \mathrm{~cm}^{-1}$, as shown in Fig. 4), which indicates a lower symmetry or a higher unit-cell $\mathrm{Z}$ number. This transition is due to the sensitivity of weak bonds against pressure. More experiments can be performed to characterize finely this transition, but this is not within the scope of our work. Simultaneously the ca. $900 \mathrm{~cm}^{-1}$ doublet merges in a single band and the $\delta\left(\mathrm{CH}_{2}\right) 1450 \mathrm{~cm}^{-1}$ and $\nu\left(\mathrm{CH}_{2}\right)$ $2900 \mathrm{~cm}^{-1}$ massifs are reorganized (see Fig. 5). The homologue palmitic acid being monoclinic at ambient pressure, a transition to a triclinic symmetry is expected. Spectra recorded at 1.9 and $3.6 \mathrm{GPa}$ are rather similar while a new mode appears at $\simeq 150 \mathrm{~cm}^{-1}$ at $5 \mathrm{GPa}$. We observe also an unexpected down shift of the ca. 180 $190 \mathrm{~cm}^{-1}$ peaks. This antagonist behaviour may be related to a loss of planarity of the CC chain, allowing thus angle modifications. The spectra also change at about $12 \mathrm{GPa}$ with a strong broadening of all the bands ; intensity modification of the $\nu(\mathrm{C}-\mathrm{C}) 1100-1200 \mathrm{~cm}^{-1}$ and $\delta(\mathrm{CH})_{2} 1450 \mathrm{~cm}^{-1}$ bands (see Fig. 6), and disappearance of the ca. $1475 \mathrm{~cm}^{-1}$ band are observed. The global behaviour is very similar to that reported for palmitic and stearic acids [37,21]. As a conclusion, three phase transitions are observed in Raman spectra, namely at $\simeq 1,5$ and $12 \mathrm{GPa}$ (see Table 3.2). The big changes observed above $1 \mathrm{GPa}$ are assigned to the destruction of the dimer H-bonding that allows a lowering of the symmetry with loss of the inversion centre imposed by the dimer structure. The large broadening and intensity change of $\nu \mathrm{C}-\mathrm{C}$ modes above $12 \mathrm{GPa}$ can be explained by a loss of the regularity/planarity of the $\mathrm{CC}$ aliphatic chain. 

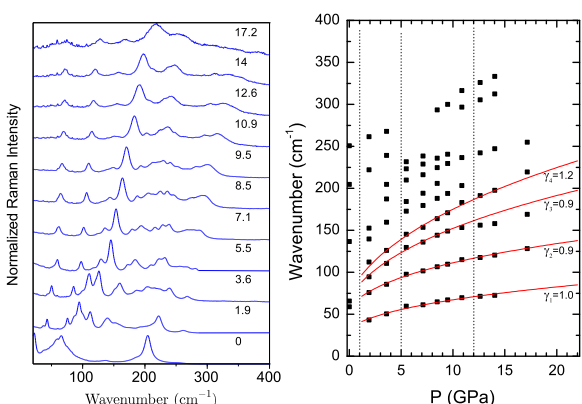

Fig. 4 (a) Raman spectra of polycristalline lauric acid for the spectral range $20-400 \mathrm{~cm}^{-1}$ recorded at different pressures (in $\mathrm{GPa}$ ). (b) Wavenumber as a function of pressure plot for the same spectral region showed in (a) up to $350 \mathrm{~cm}^{-1}$ (only major bands are shown). Red lines are obtained with Eq. 2(see text). $\gamma_{i}$ are Grüneisen values associated with each branch labelled $i$. Many modes between 50 and $180 \mathrm{~cm}^{-1}$ can be assigned to lattice modes [21].

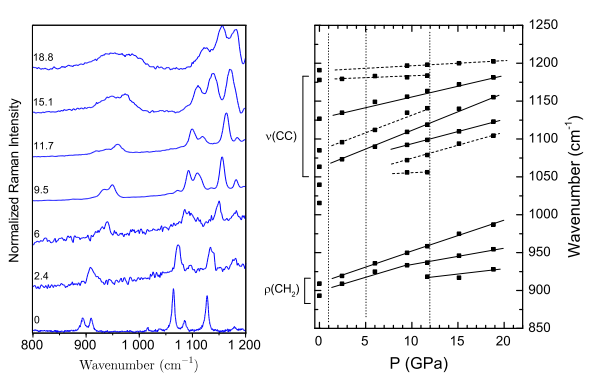

Fig. 5 (a) Raman spectra of polycrystalline lauric acid for the spectral range $800-1200 \mathrm{~cm}^{-1}$ recorded at different pressures (in $\mathrm{GPa}$ ). (b) Wavenumber as a function of pressure plot for the same spectral region showed in (a). Probable mode assignments are given at the left of the figure, as suggested by Ref. [21].

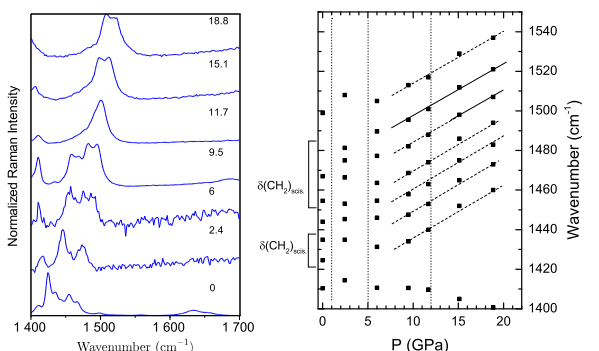

Fig. 6 (a) Raman spectra of polycrystalline lauric acid for the spectral range 1400-1700 $\mathrm{cm}^{-1}$ recorded at different pressures (in GPa). (b) Wavenumber as a function of pressure plot for the spectral region 1400-1550 $\mathrm{cm}^{-1}$. The evolution of some bands (long dash) is obtained with the help of Fig.4(b) in Ref. [21]. Probable mode assignments are given at the left of the figure, as suggested by Ref. [21]. 


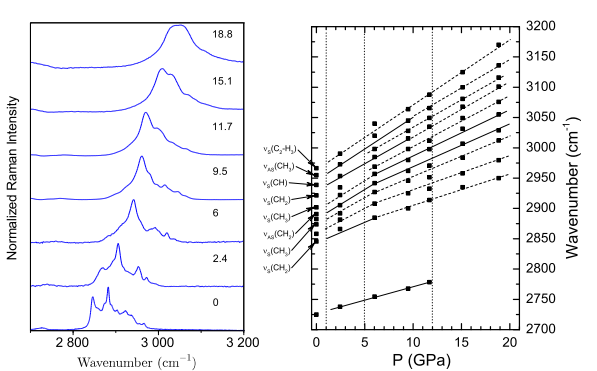

Fig. 7 (a) Raman spectra of polycrystalline lauric acid for the spectral range $2700-3700 \mathrm{~cm}^{-1}$ recorded at different pressures (in GPa). (b) Wavenumber as a function of pressure plot for the same spectral region showed in (a). Probable mode assignments are given at the left of the figure, as suggested by Ref. [21].

\subsection{Comparison between Raman scattering and picosecond acoustics}

These two techniques, low frequency Raman modes typically below $200 \mathrm{~cm}^{-1}$ (see Fig 4b.), and picosecond acoustics, provide information about elastic properties of lauric acid.

The pressure dependence of lattices Raman modes can be analyzed with the Grüneisen parameter [39] and an appropriate equation of state. The Grüneisen equation is the relation between the mode frequency $\omega$ and the volume $V$. It is defined, for mode $i$, by $\gamma_{i}=-d \ln \omega_{i} / d \ln V$. Considering $\gamma_{i}$ pressure independent, the pressure dependence of the bulk modulus of lauric acid can be described by Murnaghan equation of state [40]. In this model, the bulk modulus $B$ varies linearly with pressure such as $B(P)=B_{0}+B_{0}^{\prime} P$, where $B_{0}$ is the bulk modulus at ambient pressure, and $B_{0}^{\prime}$ is the derivative of the bulk modulus $B_{0}^{\prime}=\left(\frac{d B}{d P}\right)_{0}$ at ambient pressure. So at low wavenumbers the frequency of the mode follows the relation

$$
\omega_{i}(P)=\omega_{i}(0)\left[1+\frac{B_{0}^{\prime}}{B_{0}} P\right]^{\gamma_{i} / B_{0}^{\prime}} .
$$

A fit of the four lowest frequency modes (see Fig. $4 \mathrm{~b}$ ) gives $B_{0}=4.0 \mathrm{GPa}$ and $B_{0}^{\prime}=3.05$. The values of $\gamma_{i}$ assigned to each vibration mode are given in Fig. $4(\mathrm{~b})$.

The values of $B_{0}$ and $B_{0}^{\prime}$ are in accordance with the nature of the vibrational modes which are supposed to arise from lattice vibrations reflecting bulk properties.

At high wavenumbers, however, the evolution of the frequency can be considered linear as a function of pressure.

\section{Conclusion}

In this study we explored the vibrational properties of lauric acid submitted to high pressures up to $25 \mathrm{GPa}$. Lauric acid, submitted to high pressures above $2 \mathrm{GPa}$, exhibits a continuous transformation without structural phase transition, in addition with changes in vibrational Raman modes. Based on those findings, the next step will be dedicated to the study of the behaviour of fcc supercrystals of lauric acid coated metallic NCs, submitted to pressure. The aim will be to explore 
the stability of the lauric acid surrounding the NCs, that in turn, the stability of the supercrystal under pressure as well as, the expected phase transitions arising from a change in the interactions between NCs. The choice of lauric acid as a pressure transmitting medium to study these systems is justified by its chemical compatibility with the samples, its relative softness with respect to other PTMs, and its stability at high pressures.

\section{Acknowledgements}

The authors acknowledge Marc Morand and the "Cellule Projet" for his technical support on the picosecond acoustics set-up. Funding was provided by Labex Michem and Labex Matisse. Femtosecond laser micro-machining at the Institut de Mineralogie, de Physique des Materiaux et de Cosmochimie (IMPMC), Paris, has been developed and realized by the "Cellule Projet" with the financial support of ANR 2010-JCJC-604-01.

\section{References}

1. Younjin Min, Mustafa Akbulut, Kai Kristiansen, Yuval Golan, and Jacob Israelachvili. The role of interparticle and external forces in nanoparticle assembly. Nature materials, $7(7): 527-538,2008$.

2. Aurélien Crut, Paolo Maioli, Natalia Del Fatti, and Fabrice Vallée. Acoustic vibrations of metal nano-objects: Time-domain investigations. Physics Reports, 549:1 - 43, 2015. Acoustic vibrations of metal nano-objects: Time-domain investigations.

3. Sebastian Volz, Jose Ordonez-Miranda, Andrey Shchepetov, Mika Prunnila, Jouni Ahopelto, Thomas Pezeril, Gwenaelle Vaudel, Vitaly Gusev, Pascal Ruello, Eva M. Weig, Martin Schubert, Mike Hettich, Martin Grossman, Thomas Dekorsy, Francesc Alzina, Bartlomiej Graczykowski, Emigdio Chavez-Angel, J. Sebastian Reparaz, Markus R. Wagner, Clivia M. Sotomayor-Torres, Shiyun Xiong, Sanghamitra Neogi, and Davide Donadio. Nanophononics: state of the art and perspectives. The European Physical Journal B, 89(1):15, Jan 2016

4. Isabelle Lisiecki, Dario Polli, Cong Yan, Giancarlo Soavi, Eugène Duval, Giulio Cerullo, and Marie-Paule Pileni. Coherent longitudinal acoustic phonons in three-dimensional supracrystals of cobalt nanocrystals. Nano Letters, 13(10):4914-4919, 2013. PMID: 24010961.

5. A Courty, A Mermet, PA Albouy, E Duval, and MP Pileni. Vibrational coherence of self-organized silver nanocrystals in fcc supra-crystals. Nature materials, 4(5):395, 2005.

6. Shouheng Sun, Christopher B Murray, Dieter Weller, Liesl Folks, and Andreas Moser. Monodisperse fept nanoparticles and ferromagnetic fept nanocrystal superlattices. science, 287(5460):1989-1992, 2000.

7. An-Hui Lu, E emsp14L Salabas, and Ferdi Schüth. Magnetic nanoparticles: synthesis, protection, functionalization, and application. Angewandte Chemie International Edition, 46(8):1222-1244, 2007.

8. Zhihong Nie, Alla Petukhova, and Eugenia Kumacheva. Properties and emerging applications of self-assembled structures made from inorganic nanoparticles. Nature nanotechnology, 5(1):15, 2010.

9. Srikanth Singamaneni, Valery N Bliznyuk, Christian Binek, and Evgeny Y Tsymbal. Magnetic nanoparticles: recent advances in synthesis, self-assembly and applications. Journal of Materials Chemistry, 21(42):16819-16845, 2011.

10. Michael A. Boles, Michael Engel, and Dmitri V. Talapin. Self-assembly of colloidal nanocrystals: From intricate structures to functional materials. Chemical Reviews, 116(18):11220-11289, 2016. PMID: 27552640.

11. I Lisiecki and MP Pileni. Synthesis of well-defined and low size distribution cobalt nanocrystals: the limited influence of reverse micelles. Langmuir, 19(22):9486-9489, 2003. 
12. S. Costanzo, G. Simon, J. Richardi, Ph. Colomban, and I. Lisiecki. Solvent effects on cobalt nanocrystal synthesis - a facile strategy to control the size of co nanocrystals. The Journal of Physical Chemistry C, 120(38):22054-22061, 2016.

13. Isabelle Lisiecki, Stuart Turner, Sara Bals, M. P. Pileni, and Gustaaf Van Tendeloo. The remarkable and intriguing resistance to oxidation of $2 \mathrm{~d}$ ordered hcp co nanocrystals. a new intrinsic property. Chemistry of Materials, 21(12):2335-2338, 2009.

14. Zewei Quan, Yuxuan Wang, In-Tae Bae, Welley Siu Loc, Chenyu Wang, Zhongwu Wang, and Jiye Fang. Reversal of hall-petch effect in structural stability of pbte nanocrystals and associated variation of phase transformation. Nano letters, 11(12):5531-5536, 2011.

15. CS Yoo, H Cynn, P Söderlind, and V Iota. New $\beta$ (fcc)-cobalt to 210 gpa. Physical review letters, 84(18):4132, 2000.

16. Luisa F Cabeza, Albert Castell, CD Barreneche, A De Gracia, and AI Fernández. Materials used as pcm in thermal energy storage in buildings: a review. Renewable and Sustainable Energy Reviews, 15(3):1675-1695, 2011.

17. S Ayrinhac, M Gauthier, G Le Marchand, M Morand, F Bergame, and F Decremps. Thermodynamic properties of liquid gallium from picosecond acoustic velocity measurements. Journal of Physics: Condensed Matter, 27(27):275103, 2015.

18. Yasaka Wada, Sotoshi Simbo, and Minoru Oda. Dispersion of ultrasonic velocity in the liquid fatty acids. Journal of the Physical Society of Japan, 5(5):345-348, 1950.

19. A Dorinson, MR McCorkle, and AW Ralston. Refractive indices and densities of normal saturated fatty acids in the liquid state. Journal of the American Chemical Society, 64(12):2739-2741, 1942.

20. D.Julian McClements and Malcolm J.W. Povey. Ultrasonic analysis of edible fats and oils. Ultrasonics, 30(6):383 - 388, 1992.

21. FF De Sousa, GD Saraiva, PTC Freire, JA Lima, P Alcantara, FEA Melo, and J Mendes Filho. Pressure-induced phase transitions in palmitic acid: C form. Journal of Raman Spectroscopy, 43(1):146-152, 2012.

22. Fan Ya, Zhou Jing, Li Shuang, Guan Fu-Ying, and Xu Da-Peng. Pressure-induced phase transition in oleic acid studied by raman spectroscopy. Chinese Physics Letters, 28(11):110702, 2011.

23. Fan Ya, Zhou Jing, and Xu Da-Peng. The investigation on the pressure-induced phase transition in linoleic acid by in situ raman spectroscopy. Spectrochimica Acta Part A: Molecular and Biomolecular Spectroscopy, 129:143-147, 2014.

24. JC Chervin, B Canny, and M Mancinelli. Ruby-spheres as pressure gauge for optically transparent high pressure cells. International Journal of High Pressure Research, 21(6):305-314, 2001.

25. C. Thomsen, H. T. Grahn, H. J. Maris, and J. Tauc. Surface generation and detection of phonons by picosecond light pulses. Phys. Rev. B, 34(6):4129-4138, Sep 1986.

26. S. Ayrinhac, M. Gauthier, L. E. Bove, M. Morand, G. Le Marchand, F. Bergame, J. Philippe, and F. Decremps. Equation of state of liquid mercury to $520 \mathrm{k}$ and 7 gpa from acoustic velocity measurements. The Journal of Chemical Physics, 140(24):244201, 2014 .

27. H.-N. Lin, R.J. Stoner, H. J. Maris, and J. Tauc. Phonon attenuation and velocity measurements in transparent materials by picosecond acoustic interferometry. Journal of Applied Physics, 69(7):3816-3822, 1991.

28. Vitalyi E. Gusev and Pascal Ruello. Advances in applications of time-domain brillouin scattering for nanoscale imaging. Applied Physics Reviews, 5(3):031101, 2018.

29. M. Khafizov, J. Pakarinen, L. He, H.B. Henderson, M.V. Manuel, A.T. Nelson, B.J. Jaques, D.P. Butt, and D.H. Hurley. Subsurface imaging of grain microstructure using picosecond ultrasonics. Acta Materialia, 112:209 - 215, 2016.

30. Alexander F. Goncharov, Michel Gauthier, Daniele Antonangeli, Simon Ayrinhac, Frédéric Decremps, Marc Morand, Alexei Grechnev, S. M. Tretyak, and Yu. A. Freiman. Elasticity and poisson's ratio of hexagonal close-packed hydrogen at high pressures. Phys. Rev. B, 95:214104, Jun 2017.

31. S Klotz, J-C Chervin, P Munsch, and G Le Marchand. Hydrostatic limits of 11 pressure transmitting media. Journal of Physics D: Applied Physics, 42(7):075413, 2009.

32. Christopher A Miles, Graham AJ Fursey, and Richard CD Jones. Ultrasonic estimation of solid/liquid ratios in fats, oils and adipose tissue. Journal of the Science of Food and Agriculture, 36(3):215-228, 1985.

33. H. Mehling and L.F. Cabeza. Heat and cold storage with PCM: An up to date introduction into basics and applications. Heat and Mass Transfer. Springer Berlin Heidelberg, 2008. 
34. C Vogel-Weill and J Corset. Spectres infrarouge et raman de l'acide stéarique et d'une série d'acides gras forme c: modes de squelette, couplage des modes longitudinaux acoustiques (lam1, lam3) avec les modes dans le plan de la liaison hydrogène du dimère en dessous de $700 \mathrm{~cm}^{-1}$. Spectrochimica Acta Part A: Molecular and Biomolecular Spectroscopy, 51(13):2357 - 2377, 1995.

35. C. Vogel-Weill and A. Gruger. étude de la conformation des acides n-nonanoique, z et e-9 octadécénoiques à $90 \mathrm{k}$ par spectrométrie infrarouge et raman. partie 1. étude par spectrométrie de vibration de la conformation de l'acide n-nonanoique à $90 \mathrm{k}$. Spectrochimica Acta Part A: Molecular and Biomolecular Spectroscopy, 52(10):1297 - 1310, 1996.

36. C Vogel-Weill and A Gruger. étude de la conformation des acides n-nonanoique, $\mathrm{z}$ et e-9 octadecenoiques à $90 \mathrm{k}$ par spectrométries infrarouge et raman ii - étude de la conformation des chaines hydrocarbonées des acides z et e-9 octadecenoiques à $90 \mathrm{k}$ par spectrométrie infrarouge et raman. Spectrochimica Acta Part A: Molecular and Biomolecular Spectroscopy, 52(13):1737 - 1755, 1996.

37. F.F. de Sousa, P.T.C. Freire, G.D. Saraiva, J.A. Lima, P. Alcantara, F.E.A. Melo, and J. Mendes Filho. Pressure-induced phase transitions in stearic acid c form. Vibrational Spectroscopy, 54(2):118 - 122, 2010. A Selection of Papers Presented at the 1st Brazilian Meeting on Raman Spectroscopy (BMRS-1).

38. David R Lide et al. Crc handbook of chemistry and physics. 12J204, 1947.

39. Jean-Paul Poirier. Introduction to the Physics of the Earth's Interior. Cambridge University Press, 2000.

40. FD Murnaghan. The compressibility of media under extreme pressures. Proceedings of the National Academy of Sciences, 30(9):244-247, 1944. 